\title{
Exposure to the anthelmintic dinitroaniline oryzalin causes changes in meiotic prophase morphology and loss of synaptonemal complexes in the nematode Caenorhabditis elegans
}

\author{
Paul Goldstein* (D) \\ Department of Biological Sciences, University of Texas at El Paso, El Paso, Texas, USA \\ *Corresponding author. E-mail: drpaulgoldstein@yahoo.com
}

(Received 05 July 2021; Revised 05 September 2021; Accepted 07 September 2021)

\begin{abstract}
The anthelmintic dinitroaniline oryzalin interferes with the formation of microtubules and inhibits meiosis and mitosis in nematodes. Exposure to oryzalin resulted in deterioration in morphology of the oocytes and loss of synaptonemal complexes at meiotic prophase I. The nuclear matrix and envelope were poorly formed, and the central rachis was diminished. These results provide the basis for the loss of fecundity after treatment with the oryzalin resulting in control of parasitic nematodes.
\end{abstract}

Key words: anthelmintic; Caenorhabditis elegans; dinitroaniline; meiosis; nematode; oryzalin; synaptonemal complexes

\section{Introduction}

Oryzalin is a dinitrosulfonamide anthelmintic, which has been used as an herbicide since it interferes with plant cells by interrupting meiosis and mitosis (Morejohn et al., 1987). Dinitroanilines interfere with the stability of microtubules in cellular functions, have anthelmintic activity, and have been used as anticancer and antiparasitic drugs (Jordan \& Kamath, 2007; Sant'anna et al., 2016). Inhibition of polymerization of microtubules causes loss of meiotic chromosome segregation, which results in the formation of aneuploidy oocytes and loss of fecundity. The mode of action of oryzalin is similar to that of the anthelmintic benzimidazole, since oryzalin also binds selectively to the $\alpha$-tubulin subunit and inhibits polymerization of microtubules (Lyons-Abbott et al., 2010; Morrissette et al., 2004).

In this study, the free-living nematode Caenorhabditis elegans was exposed to oryzalin and used as a biological model to characterize changes associated with such exposure. This nematode is ubiquitous and is found in virtually all types of environments, including marine. It reproduces primarily as a selffertilizing hermaphrodite, but a small number of morphologically distinct males $(0.3 \%)$ are also present in the population (Hodgkin et al., 1979). The adult hermaphrodite has a pair of ovaries and five pairs of autosomes with two X chromosomes (2A:XX). The pachytene nuclei are arranged peripherally around a central rachis which provides for synchronous development in each specific area of the gonad. The adult male has a single testis and five pairs of autosomes, but only a single X chromosome (2A:XO; Hodgkin, 1980). Males arise from gametes that have been produced after X-chromosome nondisjunction; thus, the two sexes experience an unequal number of $\mathrm{X}$ chromosomes, similar to humans. They must compensate

\footnotetext{
(C) The Author(s), 2021. Published by Cambridge University Press. This is an Open Access article, distributed under the terms of the Creative Commons Attribution licence (http://creativecommons.org/licenses/by/4.0), which permits unrestricted re-use, distribution and reproduction, provided the original article is properly cited.
} 
for this state of aneuploidy and develop mechanisms for gene expression and dosage compensation (Goldstein, 1987).

The synaptonemal complex (SC) is a tripartite, proteinaceous structure that is found between paired chromosomes during the pachytene stage of meiosis prophase I. The structure and function of the SC has been highly conserved throughout evolution and occurs in virtually all organisms that reproduce via meiosis (von Wettstein et al., 1984). Its role is twofold: (a) maintenance of proximity of homologous chromosomal segments such that the axial cores of the chromosome become the lateral elements of the SC (Moens, 1968) and (b) regulation of ordered meiotic disjunction in which case the SC is maintained in the chiasma (Maguire, 1982). Irregular disjunction results in the formation of nonviable gametes, aneuploidy, and loss of fecundity. The presence of RNA transcription has been described during pachytene in C. elegans (Lerner \& Goldstein, 1988).

This study represents the first examination of the changes in meiotic nuclear architecture and meiotic chromosomes after exposure to oryzalin, and provides the basis for the anthelmintic control of nematodes.

\section{Materials and methods}

\subsection{C. elegans culture}

C. elegans used in this study were $\mathrm{N} 2$ wild type and were cultured as previously described (Brenner, 1974) at a constant temperature of $16^{\circ} \mathrm{C}$. Under these conditions, the average life span was 22 days. Synchronization of larvae was achieved as previously described (Porta-de-la-Riva et al., 2012). Development of the worm is rapid: egg to adult takes 3.5 days, youth and highest fertility values are observed at 4-5 days, and middle age, with decreased fertility, occurs by 11 days old.

Dinitroaniline oryzalin, at a concentration of $25 \mu \mathrm{M}$, was used in the growth medium for the worms by dissolving it in $0.1 \%(\mathrm{v} / \mathrm{v}$ ) dimethyl sulfoxide (DMSO). Ten young worms that were exposed for $4-5$ days were randomly selected and processed for electron microscopy as previously described (Goldstein \& Slaton, 1982). The concentration of DMSO had no effect on the ultrastructure of the nematodes, as was previously shown by Goldstein (2016).

\subsection{Statistics}

Ten worms were randomly selected from each of the groups: (a) control-untreated and (b) treated. In each group, the following parameters were assessed: (a) presence of the SC; (b) presence of a normal bipartite nuclear envelope (NE) that was completely contiguous with the nucleoplasm; and (c) presence of the central rachis in the germinal zone at the stage of meiosis prophase I.

Exposure of C. elegans to the anthelmintic oryzalin is correlated with the loss of SCs at meiotic prophase, loss of the contiguous NE, and loss of the central rachis in the ovary.

\begin{tabular}{|c|c|c|c|c|c|c|}
\hline & \multicolumn{2}{|c|}{$A$} & \multicolumn{2}{|c|}{ B } & \multicolumn{2}{|c|}{ C } \\
\hline & \multicolumn{2}{|c|}{ SC present } & \multicolumn{2}{|c|}{ Contiguous NE } & \multicolumn{2}{|c|}{ Rachis present at pachytene } \\
\hline & Yes & No & Yes & No & Yes & No \\
\hline CONTROL & 10 & 0 & 10 & 0 & 10 & 0 \\
\hline TREATED & 0 & 10 & 0 & 10 & 0 & 10 \\
\hline
\end{tabular}

Analysis of each of these responses separately versus treatment/control shows that there is a "positive" correlation: 
1-sample proportions test with continuity correction

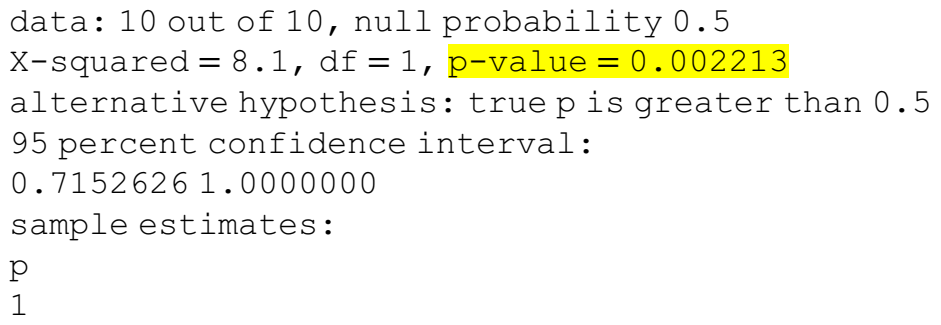

\section{Results}

The pachytene stage of meiosis is sensitive to many environmental factors. In general, nematodes require an exogenous source of sterol, since they are unable to produce their own. Thus, in the maintenance of C. elegans in culture, $5-\mu \mathrm{g} / \mathrm{ml}$ cholesterol is usually added to the medium. However, in this study, we added oryzalin, a xenoestrogen, which the worms used as the sterol source. The result of this substitution is that it links the growth of C. elegans to its ability to metabolize the oryzalin in the medium, since it is the only sterol source available for nutrition.

A brief review of the wild-type ultrastructural morphology permits comparison to the changes resulting from exposure to oryzalin. In the wild-type hermaphrodite of $C$. elegans, the pachytene nuclei are arranged peripherally around a central rachis (Figure 1). Each oocyte is connected to the

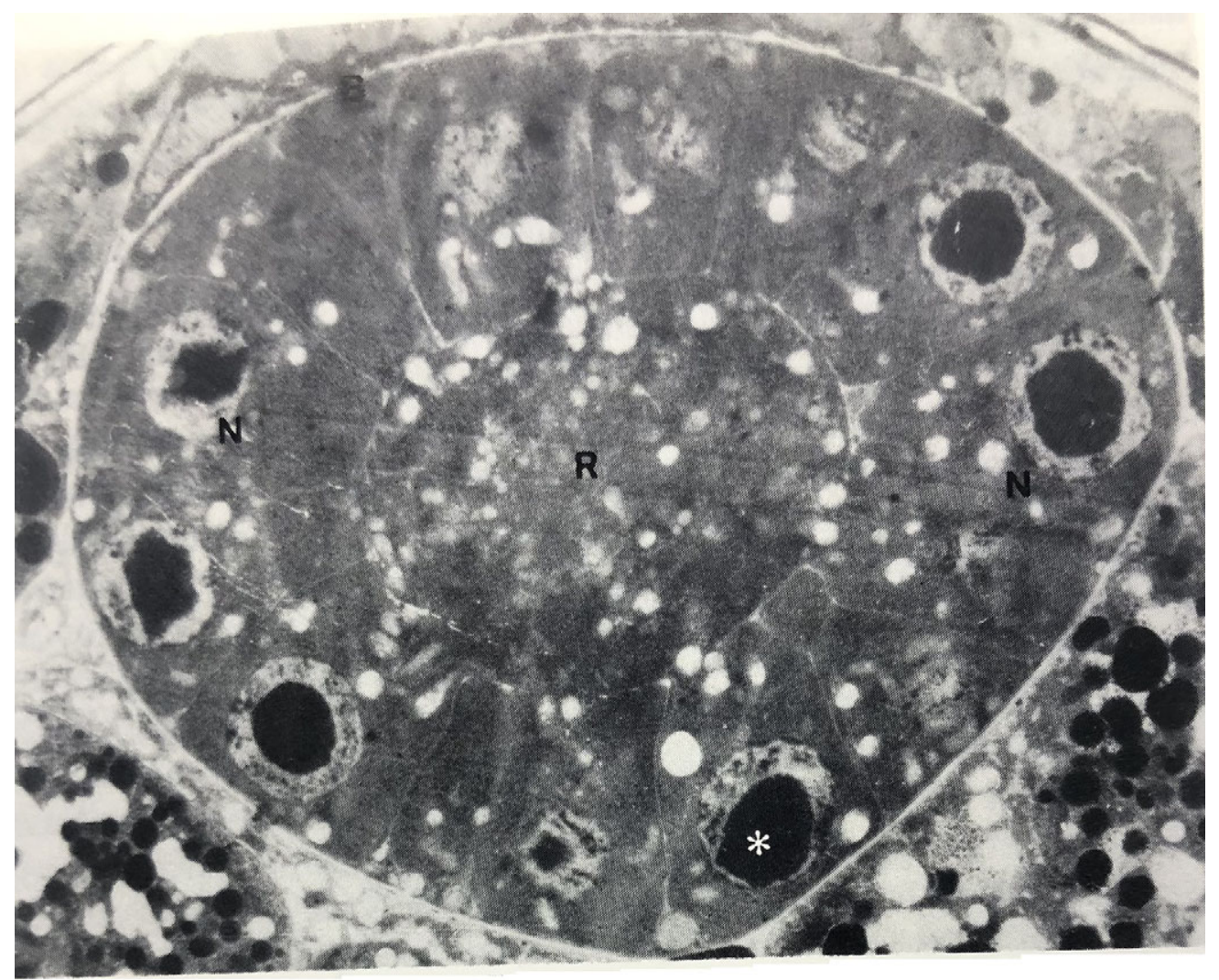

Figure 1. Nonexposed Caenorhabditis elegans. At the pachytene stage of meiotic prophase I, the nuclei $(\mathrm{N})$ are arranged peripherally around a central rachis (R). Each nucleus is connected to the rachis via a contiguous cytoplasmic bridge. Nucleolus (asterisk); Basement membrane of gonad (B). $\times 50,000$. 


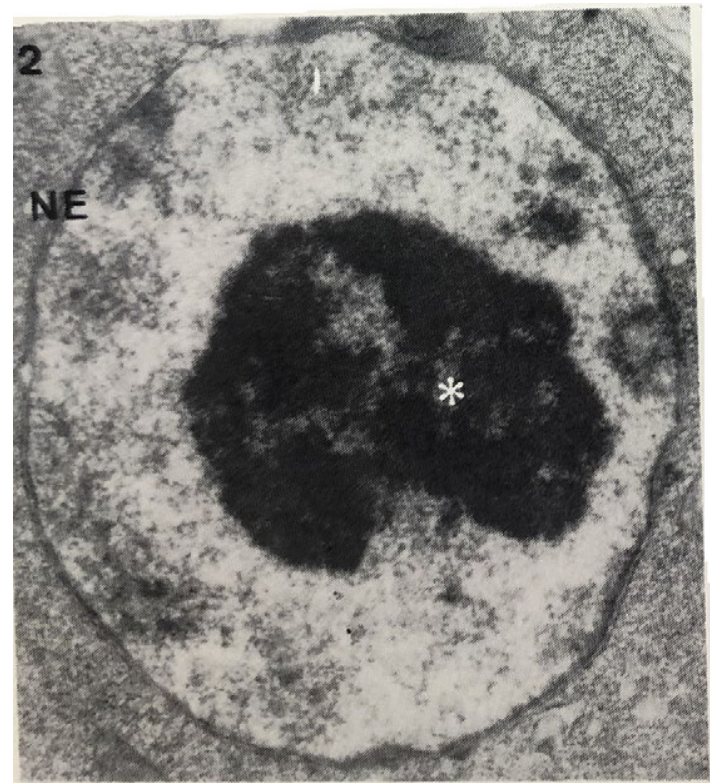

Figure 2. Nonexposed Caenorhabditis elegans. The normal nucleus at pachytene has a contiguous inner and outer nuclear envelope. Condensed chromosomes are visible within the nucleus, and the nucleolus (asterisk) is nonfragmented. $\times 25,000$.

rachis via a cytoplasmic bridge which accounts for the observed synchrony of all pachytene nuclei in that restricted area of the gonad. The peripheral arrangement of the nuclei is characteristic only of the pachytene stage, and at all other stages, the meiotic nuclei are present in a honeycomb pattern (Goldstein, 1981). Within the pachytene nucleus, a single, large, nucleolus is present, which is not fragmented and has no nucleonemata (Figure 2). SCs are present between homologously paired chromosomes (Figure 3).

After growth in $25-\mu \mathrm{M}$ oryzalin, the structure of the gonad and nucleus was severely compromised. The NE was irregular, and open spaces between the outer NE and cytoplasm were present (Figure 4). There were no SCs or any SC-related structures present. Premature manifestations of senescence were observed, such as a decrease in number of mitochondria, which are usually numerous, and increased density of the nuclear matrix (Figure 5). The arrangement of the nuclei around the central rachis was altered such that the central rachis was essentially absent and cytoplasmic bridges were destroyed (Figure 5). The bipartite NE was not completely contiguous with the nuclear plasm.

\section{Discussion}

Oryzalin, a dinitrosulfonamide, has antiparasitic activity, as has been shown in apicomplexan species (Jordan \& Kamath, 2007). It also has anthelmintic activity against nematodes (Sant'anna et al., 2016). In this study, meiosis was inhibited in the nematode $C$. elegans after exposure to oryzalin due to inhibition of microtubule polymerization. Oryzalin selectively interacts with the $\alpha$-tubulin subunits which are involved in microtubule organization. Exposure to oryzalin may result in the increase in chromosome nondisjunction, leading to increased numbers of inviable offspring.

Oryzalin causes changes in the function of the chromosomes, which result in inviable gametes. The response of chromosomes to oryzalin is drastic: alterations in DNA synthesis, breakage, loss of movement, failure to pair during meiosis, and failure to segregate during meiotic and mitotic divisions. This change in function of DNA is related to changes in DNA structure such that oryzalin has been 


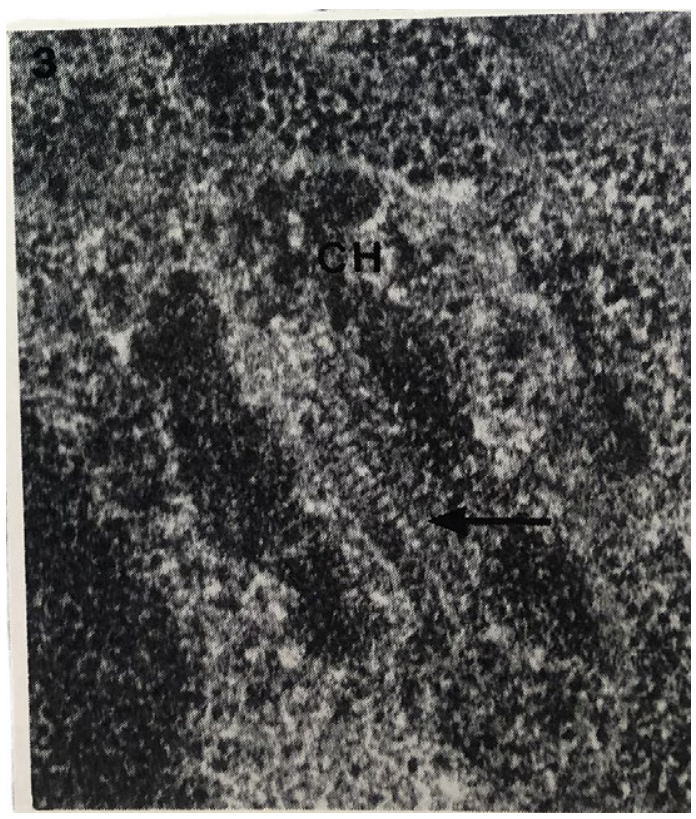

Figure 3. Nonexposed Caenorhabditis elegans. Tripartite synaptonemal complexes (SCS) are formed between homologous chromosomes during pachytene. Central element (arrow); The width of the SC is $100 \mathrm{~nm}$, Condensed Chromatin $(\mathrm{CH}) \times 15,000$.

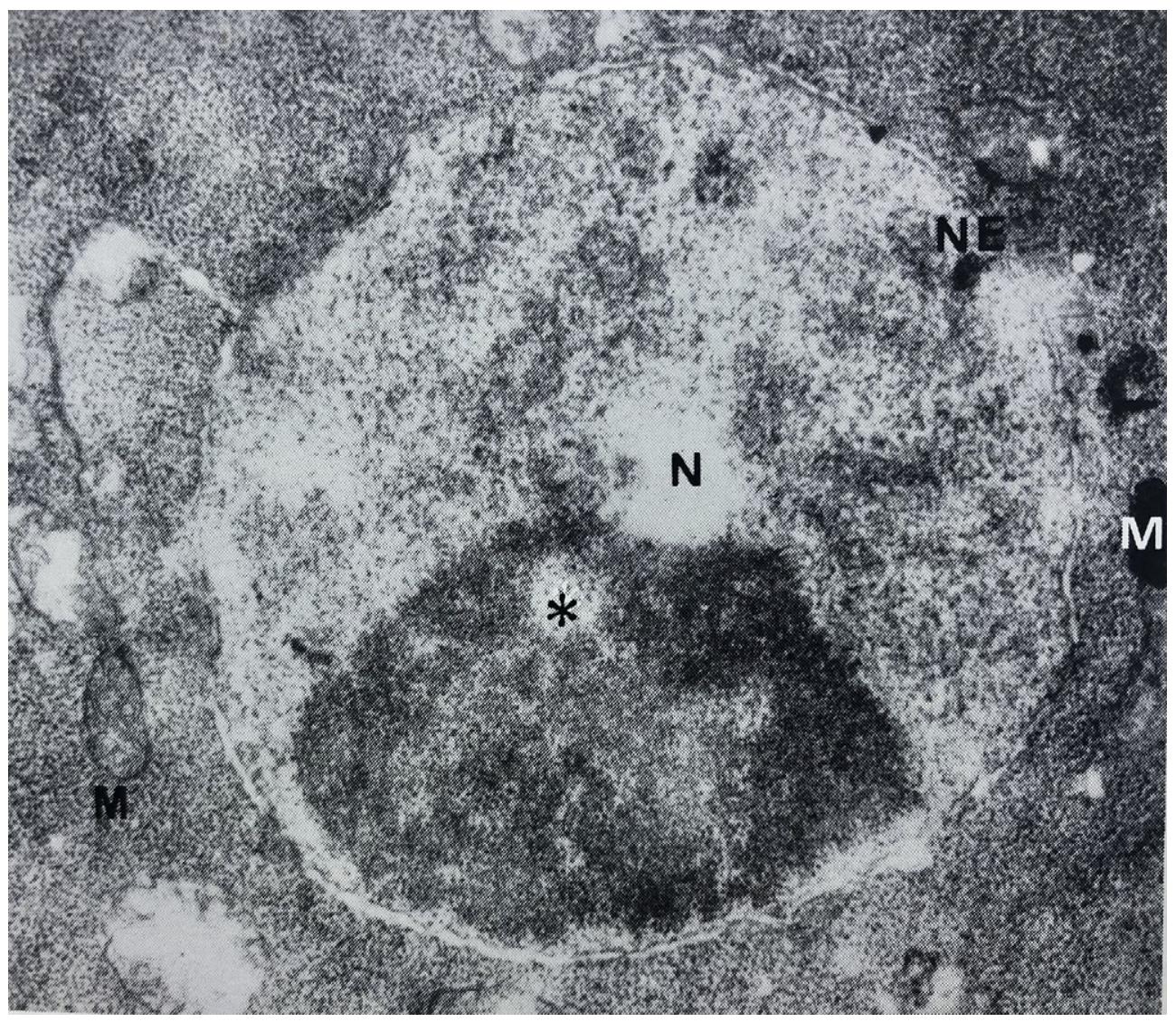

Figure 4. Nucleus from Caenorhabditis elegans after exposure to $25-\mu \mathrm{M}$ oryzalin. The nuclear matrix is compromised such that $\mathrm{SCs}$ are absent and the nucleolus (asterisk) has a nucleolar vacuole (N) associated with it. The nuclear envelope is not contiguous with the cytoplasm. (M) Mitochondrion. $\times 35,000$. 


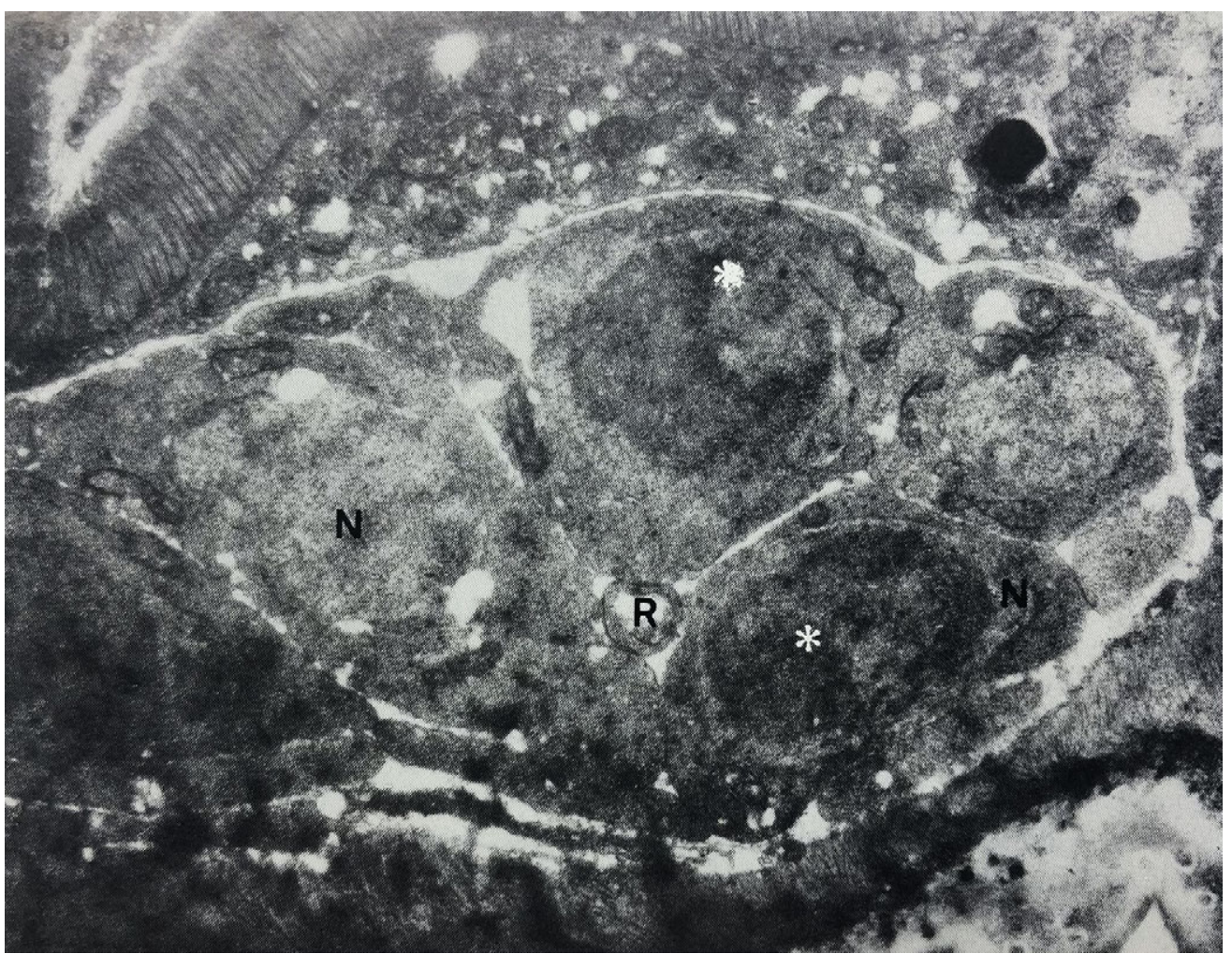

Figure 5. After exposure to $25-\mu \mathrm{M}$ oryzalin, there was disorganization of the gonad in Caenorhabditis elegans. The central rachis $(R)$ was diminished and the cytoplasmic bridges connecting the oocytes to the rachis were not present. (N) Interphaseappearing nucleus. $\times 15,000$.

shown to be mutagenic in sister chromatid exchange and alters the DNA repair mechanism (Sankula \& Khan, 2008). The secondary effects of changes in DNA structure are manifested by changes in expression and production of enzymes. Such a change may be responsible for the lack SC formation in nuclei exposed to oryzalin. It is at early stages of meiotic prophase that specific DNA replication occurs (zygotene-DNA and pachytene-DNA) which code for enzymes required for breakage and reunion of the chromosomes during crossing-over (Stern \& Hotta, 1977). If the production or activities of these enzymes are inhibited, the structure of the SC may be affected, and recombination may be terminated.

Absence of SCs may also be the result of the colchicine-like effects of oryzalin, since it has been shown that cells subjected to colchicine at premeiotic interphase have greatly decreased levels of recombination and incomplete pairing. Chromosomes, which are not fully paired during meiosis, result in changes in genic balance, which can lead to death of germ cells (Burgoyne \& Baker, 1984). In C. elegans, the effect is more pronounced, because the chromosomes are very short. The colchicine-like effect of oryzalin may significantly disrupt the segregation of meiotic chromosomes, since microtubules are not formed (as shown in Figures 11 and 15a from Goldstein, 1978). The lack of an SC in worms exposed to oryzalin may result in univalent or polyvalent formation, with subsequent formation of unbalanced gametes and loss of fecundity.

Aberrations in the NE were present in the pachytene nuclei after exposure to oryzalin. The highly hydrophobic compound oryzalin (Hugdahl \& Morejohn, 1993) interferes with the ordered structure of membranes in a similar way to that of detergents by rupturing membrane structures. Oryzalin interacts 
with hydrophobic domains of soluble and membrane-bound proteins. Production of inviable gametes is also the result of the diminished ability of nuclear-cytoplasmic interactions.

Nuclear changes occurred after exposure to oryzalin, and the synchrony of nuclei at meiotic prophase was lost, that is, interphase-appearing nuclei were observed in the pachytene region of the gonad (Figure 5). The cytoplasmic bridges that connect the oocyte to the central rachis play a major role in the synchrony of all cells in the pachytene region. Within these bridges, microtubules are also present (Foor, 1967), but in oryzalin-exposed worms, the microtubules and rachis were severely disintegrated. This would result in the loss of synchrony in the production of eggs.

\section{Conclusion}

The results of this study show that oryzalin has anthelmintic activity on the nematode C. elegans, by causing severe morphological and ultrastructural alterations in meiotic oocyte nuclei and chromosomes.

Conflict of interest. The author declares no competing interests exist.

Data availability statement. The author confirms that all the data supporting the findings of this study are available within the article.

\section{References}

Brenner, S. (1974). The genetics of Caenorhabditis elegans. Genetics, 77, 71-94.

Burgoyne, P., \& Baker, T. (1984). Meiotic pairing and gametogenic failure. In C. Evans \& H. Dickinson (Eds.), Controlling events in meiosis (pp. 349-369). Company of Biologists.

Foor, E. (1967). Ultrastructural aspects of oocyte development and shell formation in Ascaris lumbricoides. The Journal of Parasitology, 53, 1245-1261.

Goldstein, P. (1978). Ultrastructural analysis of sex determination in Ascaris lumbricoides var. suum. Chromsoma, 66, 59-69.

Goldstein, P. (1981). Sex determination in nematodes. In B. Zuckerman \& R. Rhode (Eds.), Plant-parasitic nematodes (Vol. 3 , pp. 37-60). Academic Press.

Goldstein, P. (1987). Aneuploidy in the normal life cycle of the nematode Caenorhabditis elegans. In B. Vig \& A. Sandberg (Eds.), Aneuploidy, Part A: Inclusive and etiology (pp. 187-204). Alan R. Liss.

Goldstein, P. (2016). Dimethyl sulfoxide-suppressed RNA transcription during meiosis in wild-type hermaphrodite Caenorhabditis elegans and loss of fertility. International Journal for Biological \& Pharmaceutical Research, 1, 18-22.

Goldstein, P., \& Slaton, D. (1982). The synaptonemal complexes of Caenorhabditis elegans: Comparison of wild-type and mutant strains and pachytene karyotype analysis of wild-type. Chromosoma, 84, 585-597.

Hodgkin, J., et al. (1979). Nondisjunction mutants of the nematode Caenorhabditis elegans. Genetics, 91, 67-94.

Hodgkin, J. (1980). More sex determination mutants of Caenorhabditis elegans. Genetics, 96, 649-664.

Hugdahl, J., \& Morejohn, L. (1993). Rapid and reversible high-affinity binding of the dinitroaniline herbicide oryzalin to tubulin from Zea mays. Plant Physiology, 102, 725-740.

Jordan, M., \& Kamath, K. (2007). How do microtubule-targeted drugs work? An overview. Current Cancer Drug Targets, 7 , $730-742$.

Lerner, K., \& Goldstein, P. (1988). Electron microscopic autoradiographic analysis: Evidence of RNA transcription along pachytene chromosomes of RAD-4, HIM-4 and wild-type Caenorhabditis elegans. Cytobios, 55, 51-61.

Lyons-Abbott, S., et al. (2010). $\alpha$-Tubulin mutations alter oryzalin affinity and microtubule assembly properties to confer dinitroaniline resistance. Eukaryotic Cell, 9, 1825-1834.

Maguire, M. (1982). Evidence for separate genetic control of crossing over and chiasma maintenance in maize. Chromosoma, 65, 173-178.

Moens, P. (1968). The structure and function of the synaptonemal complex in Lilium longiflorum sporocytes. Chromosoma, 28, $1-25$.

Morejohn, L., et al. (1987). Oryzalin, a dinitroaniline herbicide, binds to plant tubulin and inhibits microtubule polymerization in vitro. Planta, 172, 252-264.

Morrissette, A., et al. (2004). Dinitroanilines bind alpha-tubulin to disrupt microtubules. Molecular Biology of the Cell, 15, 1960-1968.

Porta-de-la-Riva, M., et al. (2012). Basic Caenorhabditis elegans methods: Synchronization and observation. Journal of Visualized Experiments, 64, e4019. https://doi.org/10.3791/4019

Sankula, S., \& Khan, F. (2008). Risks of oryzalin use to federally threatened California red-legged frog; Pesticide Effects Determination; Environmental Fate and Effects Division, pp. 1-133. 
Sant'anna, V., de Souza, W., \& Vommaro, R. (2016). Anthelmintic effect of herbicidal dinitroanilines on the nematode model Caenorhabditis elegans. Experimental Parasitology, 167, 43-49.

Stern, H., \& Hotta, Y. (1977). Biochemistry of meiosis. Philosophical Transactions of the Royal Society B: Biological Sciences, 277, 277-294.

Von Wettstein, D., et al. (1984). The synaptonemal complex in genetic segregation. Annual Reviews of Genetics, 18, $331-413$.

Cite this article: Goldstein P (2021). Exposure to the anthelmintic dinitroaniline oryzalin causes changes in meiotic prophase morphology and loss of synaptonemal complexes in the nematode Caenorhabditis elegans. Experimental Results, 2, e38, 1-13. https://doi.org/10.1017/exp.2021.19 


\title{
Peer Reviews
}

\author{
Reviewing editor: Dr. Z. Onur Caliskaner
}

Uskudar University, Istanbul, Turkey, 34662

This article has been accepted because it is deemed to be scientifically sound, has the correct controls, has appropriate methodology and is statistically valid, and has been sent for additional statistical evaluation and met required revisions.

doi:10.1017/exp.2021.19.pr1

Review 1: Exposure to the Anthelmintic Dinitroaniline Oryzalin Causes Changes in Meiotic Prophase Morphology and Loss of Synaptonemal Complexes in the Nematode Caenorhabditis elegans

\section{Reviewer: Dr. Huanyu Qiao (D)}

University of Illinois System, Comparative Biosciences, Department of Comparative Biosciences, 2001 South Lincoln Ave, M/C 002, Urbana, Illinois, United States, 61802

Date of review: 18 August 2021

\begin{abstract}
(C) The Author(s), 2021. Published by Cambridge University Press. This is an Open Access article, distributed under the terms of the Creative Commons Attribution licence (http://creativecommons.org/licenses/by/4.0), which permits unrestricted re-use, distribution and reproduction, provided the original article is properly cited.
\end{abstract}

Conflict of interest statement. Reviewer declares none.

\section{Comment}

Comments to the Author: Reviewer comments:

In this manuscript, the author discussed the effects of oryzalin on Caenorhabditis elegans. The author treated C. elegans with $25 \mathrm{uM}$ of oryzalin and examine the worm sections under electron microscopy. They found that oryzalin could cause morphological changes and synaptonemal-complex (SC) loss. The nuclear matrix and envelope are abnormal and the central rachis disappears. The result of this paper is only supported by electron microscopy and only one dose of oryzalin is used. It is hard to draw a conclusion based on their data and more evidence would be needed.

Major points:

1. The methodology of this manuscript is a big concern. C. elegans is like a bag of oocytes. Meiocytes are located in different zones along the worm body, including transitional zone, pachytene zone, diplotene zone, and diakinesis zone. Immunostaining is easy to identify different stages and monitor any synapsis problems. However, only EM was used in this manuscript. The author used central rachis to identify the pachytene stage, which is absent in the treated worms. How does the author know he/she/ they compare the treated and untreated worms at the same pachytene stage? If the staging is wrong or the oryzalin altered the oocyte development, the fundamental results/discovery of this manuscript does not make sense.

2. Many data should be quantified in a graph, for example, the number of mitochondria could be counted and presented in a bar chart.

3. The author should specify why they chose $25 \mathrm{uM}$ oryzalin instead of other concentrations. What is the environmentally relevant dose? Is there a dose-dependent effect? 
4. The author should present a SC image of the treatment group corresponding to figure 3. Do the SCs still have axial elements?

5. DNA replication completes before pachytene stage and happens in pre-meiotic $S$ phase. There should be no pachytene DNA replication. In general, the literature cited in this paper is old.

6. The discussion about colchicine is confusing. Colchicine is a drug that can disrupt spindle formation and is not directly related to homologous recombination and pairing. The duration of oryzalin treatment is too long (4-5 days). A shorter treatment can help the author pinpoint when the defects happen?

Minor points:

1. The author showed the results on oocytes. What about the spermatocytes? Do they have similar SC defects as oocytes?

2. In figure 4 , the author has stated "decrease in number of mitochondria and increased density of the nuclear matrix", "nuclear envelope (NE) is not contiguous". The author should use arrows to point them out in the figures.

3. Abstract, line 4: meiosis prophase I

4. Introduction, paragraph 1 , line 1 : since in? reword the sentence.

5. Introduction, paragraph 1, line 3-5: rephase the sentence.

6. Introduction, paragraph 1 , line 8 : what does "it" refer to?

7. Introduction, paragraph 3 , line 2 : meiosis prophase I

8. Introduction, paragraph 3, line 2: Which aspects of the SCs are conserved? Function or structure or both?

9. Materials and Methods: paragraph 1, line 3: and hermaphrodites?

10. Results, paragraph 1, line 5-7: the point needs to be clarified.

11. Figures 1-3: can be combined to figure $1 \mathrm{a}, \mathrm{b}, \mathrm{c}$

12. Figure 1-4: no scale bars, figure legends need more details.

13. Figure 1: meiosis prophase I

14. Discussion, paragraph 2, line 8: the formation of

15. Discussion, paragraph 2, line 7-11: the sentence is too long and the statement is wrong

16. Discussion, paragraph 3, line 4: the death of

17. Discussion, paragraph 3, line 9-11: two "result in" in one sentence

\section{Score Card}

Presentation

2.7

Is the article written in clear and proper English? (30\%)

Is the data presented in the most useful manner? (40\%)

Does the paper cite relevant and related articles appropriately? (30\%)

Context

Does the title suitably represent the article? $(25 \%)$

Does the abstract correctly embody the content of the article? (25\%)

Does the introduction give appropriate context? (25\%)

Is the objective of the experiment clearly defined? (25\%) 
Analysis

2.6

Does the discussion adequately interpret the results presented? (40\%)

Is the conclusion consistent with the results and discussion? (40\%)

Are the limitations of the experiment as well as the contributions of the experiment clearly outlined? (20\%) 


\section{Review 2: Exposure to the Anthelmintic Dinitroaniline Oryzalin Causes Changes in Meiotic Prophase Morphology and Loss of Synaptonemal Complexes in the Nematode Caenorhabditis elegans}

Reviewer: Dr. Aimee Jaramillo-Lambert Ph.D.

University of Delaware, Biological Sciences, 105 The Green, 118 Wolf Hall, Newark, Delaware, United States, 19716-5600

Date of review: 03 September 2021

(C) The Author(s), 2021. Published by Cambridge University Press. This is an Open Access article, distributed under the terms of the Creative Commons Attribution licence (http://creativecommons.org/licenses/by/4.0), which permits unrestricted re-use, distribution and reproduction, provided the original article is properly cited.

Conflict of interest statement. Reviewer declares none.

\section{Comment}

Comments to the Author: The author very nicely demonstrated that the anthelmintic drug oryzalin causes changes to the structure of the $\mathrm{C}$. elegans germ line and disrupts meiosis. However, some conclusions drawn in the discussion do not reflect the results presented. 1). The author states that there is a "loss of cytoplasmic components." What, specifically, are these components? All listed should be pointed out in the control. 2) The author states that the experimental treatment resulted in inhibition of microtubule polymerization and loss of kinetochores on the chromosomes. These results were not presented in the included figures (wild-type or treated worms). 3) The final paragraph states that a loss of synchrony was observed during oryzalin treatment with some interphase-looking nuclei. Please point this out in the figures provided. 4) Results says that there is a decrease in the number of mitochondria. How was this determined? 5) Fig. 5-Are the nuclei enlarged? Are there fewer nuclei compared to WT? 6) Methods-How were the worms staged? Cite source for timing of development. 7) Second paragraph of the discussion, I am unclear on what the author is trying to present here. Please revise to clarify the link between oryzalin, DNA replication, recombination, and protein production. Minor comments that need to be addressed: 1) Labeling of the figures is really hard to see (e.g. I cannot see the arrow or the label CH in Fig. 3). 2) Is it possible to point out the cytoplasmic bridges in Fig 1? 3) Fig. 4-what is the "M" label?

\section{Score Card}

Presentation

3.6

Is the article written in clear and proper English? (30\%)

Is the data presented in the most useful manner? (40\%)

Does the paper cite relevant and related articles appropriately? (30\%)

\section{Context}

Does the abstract correctly embody the content of the article? (25\%)

Does the introduction give appropriate context? (25\%)

Is the objective of the experiment clearly defined? (25\%) 
Analysis

2.8

Does the discussion adequately interpret the results presented? (40\%)

Is the conclusion consistent with the results and discussion? (40\%)

Are the limitations of the experiment as well as the contributions of the experiment clearly outlined? (20\%) 